\title{
Intracranial hemorrhage from dural arteriovenous fistulas: clinical features and outcome
}

\author{
David J. Daniels, M.D., Ph.D., ${ }^{1}$ Ananth K. Vellimana, M.D., ${ }^{2}$ Gregory J. Zipfel, M.D., ${ }^{2}$ \\ AND GiUSEPPe LANZino, M.D. ${ }^{1}$
}

${ }^{1}$ Department of Neurological Surgery, Mayo Clinic, Rochester, Minnesota; and ${ }^{2}$ Department of Neurosurgery, Washington University in St. Louis, Missouri

\begin{abstract}
Object. In this paper the authors' goal was to review the clinical features and outcome of patients with intracranial dural arteriovenous fistulas (DAVFs) who presented with hemorrhage.

Methods. A retrospective study of 28 patients with DAVFs who presented with intracranial hemorrhage to 2 separate institutions was performed. The information reviewed included clinical presentation, location and size of hemorrhage, angiographic features, treatment, and clinical and radiologically documented outcomes. Clinical and radiological follow-up were available in 27 of 28 patients (mean follow-up 17 months).

Results. The vast majority of patients were male (86\%), and the most common presenting symptom was suddenonset headache. All DAVFs had cortical venous drainage, and about one-third were associated with a venous varix. The most common location was tentorial (75\%). Treatment ranged from endovascular (71\%), surgical (43\%), Gamma Knife surgery (4\%), or a combination of modalities. The majority of fistulas $(75 \%)$ were completely obliterated, and most patients experienced excellent clinical outcome (71\%, modified Rankin Scale score of 0 or 1). There were no complications in this series.

Conclusions. Case series, including the current one, suggest that the vast majority of patients who present with intracranial hemorrhage from a DAVF are male. The most common location for DAVFs presenting with hemorrhage is tentorial. Excellent outcomes are achieved with individualized treatment, which includes various therapeutic strategies alone or in combination. Despite the hemorrhagic presentation, almost two-thirds of patients experience a full recovery with no or minimal residual symptoms.

(http://thejns.org/doi/abs/10.3171/2013.4.FOCUS1335)
\end{abstract}

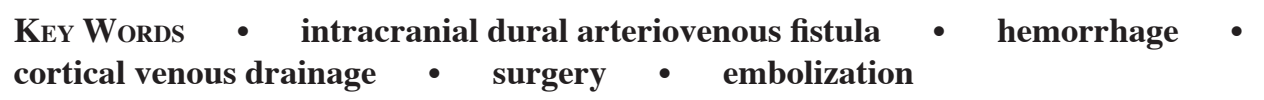

I NTRACRANIAL hemorrhage is one of the most serious clinical manifestations of intracranial DAVFs..$^{1,6-9,11}$ Many studies have focused on risk factors for aggressive clinical behavior. ${ }^{1,4,14}$ However, there are no series that have specifically evaluated the clinical characteristics and outcome of patients who suffered an intracranial hemorrhage as presenting manifestation of a DAVF., $, 6,11,13$ In this study, we analyze clinical features and outcome of 28 patients with intracranial hemorrhage from a DAVF who were evaluated and treated at 2 institutions.

\section{Methods}

Institutional review board approval from both the Mayo Clinic and Washington University in St. Louis was

\footnotetext{
Abbreviations used in this paper: DAVF $=$ dural arteriovenous fistula; GKS = Gamma Knife surgery; $\mathrm{mRS}=$ modified Rankin Scale.
}

received for this study. We retrospectively reviewed our institutions' patient databases (from 2008 to 2012, Mayo Clinic, and from 1996 to 2012, Washington University in St. Louis) and identified 28 patients (mean age 56.5 years) who presented with hemorrhage from a DAVF. These patients are the focus of this report. Information collected included demographics (age and sex), symptoms at presentation, Cognard classification (Table 1), location of the fistula, extension of hemorrhage (intraparenchymal, subdural, subarachnoid, or intraventricular), treatment modality, and final radiological and clinical follow-up. Fistulas were localized to the tentorium if the vein draining the intradural nidus emerged from the superior or inferior tentorial surface, the area of the tentorial incisura, including the galenic system, and the tentorial attachment. Thus, fistulas draining through the petrosal complex were included in the "tentorial" DAVFs as lateral tentorial rather than superior petrosal sinus fistulas in accordance with the classification system of Lawton et al. ${ }^{8}$ 
TABLE 1: Cognard classification scheme of intracranial DAVFs

\begin{tabular}{ccl}
\hline Cognard Type & No. of Patients $(\%)$ & \multicolumn{1}{c}{ Description* $^{*}$} \\
\hline I & 0 & main sinus w/ anterograde flow \\
Ila & 0 & main sinus w/ retrograde flow into sinus \\
Ilb & $5(18)$ & main sinus w/ retrograde flow into cortical veins \\
Ila+b & 0 & main sinus w/ retrograde flow into cortical veins \& main sinus \\
III & $12(43)$ & direct cortical venous drainage w/o varix \\
IV & $10(36)$ & direct cortical venous drainage w/ venous varix \\
V & $1(4)$ & Type IV w/ spinal drainage \\
\hline
\end{tabular}

* Classification based on Cognard et al.

\section{Results}

Nineteen $(68 \%)$ of the 28 patients presented with headache from intracranial hemorrhage, and most patients were male (86\%). The clinical signs and symptoms in these patients are summarized in Table 2. Tentorial fistulas $(75 \%)$ were the most common type of DAVF followed by the transverse-sigmoid DAVF location. Intraparenchymal hemorrhage $(71 \%)$ was the most common bleeding pattern identified with a mean volume of $11 \mathrm{ml}$ per patient and was most often near the fistula rather than distant from the actual point of fistulization. Other hemorrhage extensions include intraventricular (32\%), subarachnoid (21\%), and subdural (18\%). All fistulas had cortical venous drainage (Cognard IIb or greater) and more than one-third (36\%) were associated with a venous varix (Cognard Grade IV).

Twenty-six patients underwent treatment for their

TABLE 2: Demographics, location of fistula, and extent of presenting hemorrhage

\begin{tabular}{lc}
\hline \multicolumn{1}{c}{ Parameter } & Value* \\
\hline no. of patients & 28 \\
mean age in yrs & 56.5 \\
male & $24(86)$ \\
presenting symptom/sign & \\
headache & $19(68)$ \\
altered mental status/coma & $6(21)$ \\
seizures & $3(11)$ \\
DAVF grade & \\
cortical venous drainage & $28(100)$ \\
varix & $10(36)$ \\
location of fistula & \\
tentorial & $21(75)$ \\
transverse sinus & $5(18)$ \\
other & $2(7)$ \\
hemorrhage & \\
intraparenchymal & $20(71)$ \\
intraventricular & $9(32)$ \\
subarachnoid & $6(21)$ \\
subdural & $5(18)$ \\
\hline
\end{tabular}

* Values are the number of patients (\%) unless noted otherwise. fistula (Table 3). Twenty patients were treated using endovascular techniques (transarterial or transvenous or both) as the first treatment option with the goal of cure or in preparation (adjunct) to other therapeutic modalities. Seven patients underwent various therapeutic combinations either as planned therapeutic strategy or because of failure of the initial therapeutic strategy to completely obliterate the DAVF. About half (43\%) of the patients underwent surgery (either as initial treatment or for failed endovascular treatment), and $25 \%$ underwent combined therapy. Of the 26 patients treated, 21 patients (75\% of all patients) had complete obliteration of their fistula after treatment or at follow-up (mean length of radiographic follow-up 17 months).

Clinical outcomes were good, with $71 \%$ of patients reporting mRS scores of either 0 or 1 (no symptoms or minimal residual symptoms). No complications occurred during or after treatment. Figure 1 illustrates an index case.

\section{Discussion}

In a contemporary series of 28 consecutive patients with intracranial hemorrhage from a DAVF, we found that 1) despite the hemorrhagic presentation, $71 \%$ of patients achieved an excellent clinical outcome (mRS score of 0 or 1$)$; 2 ) the vast majority $(86 \%)$ of patients were males; 3) intraparenchymal hemorrhage was the most common

TABLE 3: Treatment modality and radiological and clinical outcomes

\begin{tabular}{lc}
\hline \multicolumn{1}{c}{ Parameter } & Value $^{*}$ \\
\hline treatment & \\
endovascular & $20(71)$ \\
surgery & $12(43)$ \\
GKS & $1(4)$ \\
combination & $7(25)$ \\
complete radiographic obliteration & $21(75)$ \\
mean follow-up in mos & 17 \\
mRS score & \\
$0-1$ & $20(71)$ \\
$2-3$ & $2(7)$ \\
$4-5$ & $3(11)$ \\
\hline
\end{tabular}

* Values are the number of patients (\%) unless noted otherwise. 

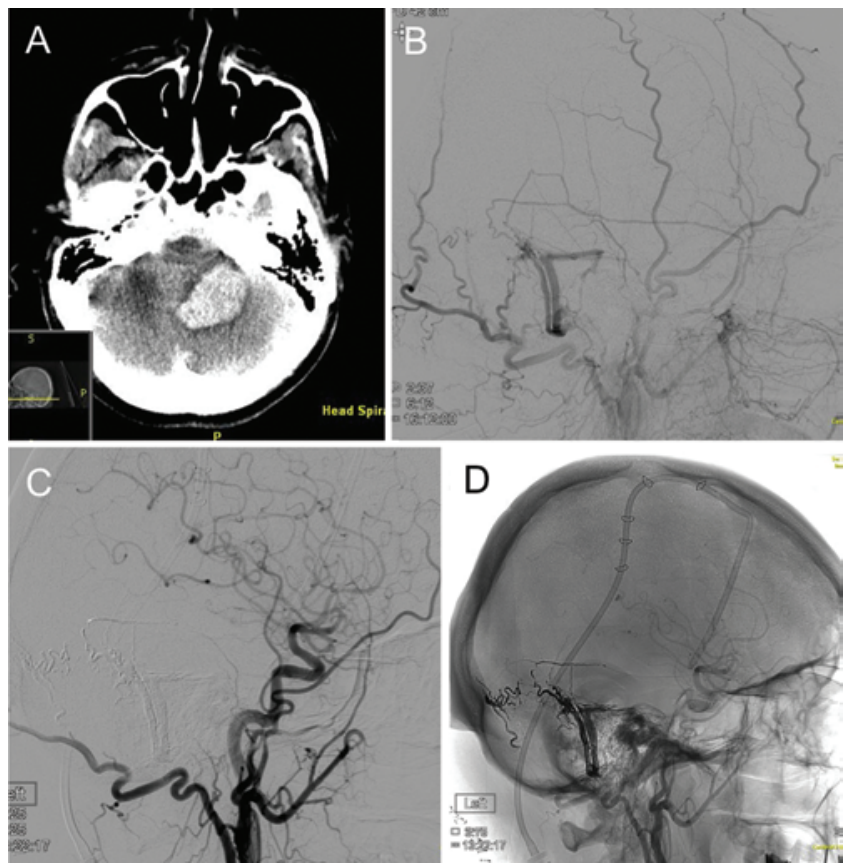

FIG. 1. A 67-year-old man developed the worst headache of his life followed by a rapid coma. A: Non-contrast enhanced head CT showing a large left cerebellar hemorrhage. B: Left external carotid artery digital subtraction angiogram, lateral projection, showing a DAVF of the sigmoid sinus fed by the posterior branch of the middle meningeal artery and occipital artery with retrograde cortical venous drainage and a venous varix. C: Left carotid artery digital subtraction angiogram, lateral projection, obtained after Onyx embolization, showing complete obliteration of the fistula. D: Left carotid artery nonsubtracted angiogram, lateral projection, Onyx cast following treatment. Despite a long hospital course, the patient made a good clinical recovery with residual postural vertigo as the only residual symptom/sign.

pattern of bleeding ( $71 \%$ of cases) with its epicenter more frequently centered around the point of fistulization rather than distal to it; and 4) an individualized therapeutic approach using various strategies alone or in combination achieved a high rate of complete obliteration with very low complication rates.

Despite the recognition of hemorrhage as one of the most serious sequelae of intracranial DAVFs, to our knowledge, there are no large series focusing on clinical characteristics and outcome of patients with DAVFs who specifically present with hemorrhage. The observation that most patients can recover after a hemorrhage from an intracranial DAVF is similar to that reported in patients with hemorrhage from parenchymal arteriovenous malformations. ${ }^{2}$ This prognosis is decidedly better than that reported for ruptured aneurysms. It is possible that our series reflects patients evaluated and treated at 2 tertiary referral centers with a referral bias, and we may have missed patients who were not transferred because of very poor clinical condition. However, our data are in agreement with the observation of Piippo and coworkers ${ }^{11}$ from a "stable" and fixed catchment area of 2 neurosurgical departments in Finland. During a 60-year interval, 261 DAVFs in 251 patients were evaluated at these centers; 34 patients (14\%) presented with hemorrhage. Although no separate outcome data were presented for these patients, none of these 34 patients was re- ported to be comatose at presentation, underscoring the often relatively good clinical condition encountered in these cases. The more benign clinical course of hemorrhage due to a DAVF compared with patients with other vascular lesions such as intracranial aneurysms is most likely related to the bleeding site being venous (although arterialized) rather than from a direct arterial source. ${ }^{6}$

The vast majority $(86 \%)$ of patients in our series were male. A male predominance in patients with DAVFs presenting with hemorrhage was also evident in other series. In the Finnish series of 261 DAVFs, men were more likely than women to present with hemorrhage. ${ }^{11}$ In that study, hemorrhagic presentation was found in 23 (21\%) of 112 men as opposed to only $11(8 \%)$ of 139 women. The different propensity to hemorrhage according to sex is likely the result of the different distribution of intracranial DAVFs between men and women. Mitsuhashi and coworkers ${ }^{10}$ reported a 32:9 male-to-female ratio in patients with dural shunts located in the "lateral epidural" space, suggesting that these fistulas may have a different etiopathogenesis and clinical features from DAVFs in other more common locations, such as the cavernous sinus or the transverse sinus, which often show a female predominance or no sex predominance, respectively. ${ }^{5}$ Further investigation of these differences may indeed shed more light into a possible different etiology of this subtype of DAVFs.

With modern endovascular techniques, better understanding of the pathophysiology and anatomy of intracranial DAVFs, individualized treatment results in high rates of complete obliteration with a low risk to the patient. For DAVFs presenting with hemorrhage, we prefer a therapeutic strategy with high chance of cure and complete angiographic obliteration. With the advent of newer embolic agents (Onyx, Covidien), we have been able to safely and effectively obliterate many tentorial DAVFs. ${ }^{12}$ In our experience, a sine qua non for successful embolization is the presence of a well-developed posterior branch of the middle meningeal artery supplying the lesion. This branch is relatively straight and allows distal catheterization, which in turn increases the likelihood of penetration of the embolic agent well into the proximal draining vein. If embolization is not feasible as a definitive therapy or fails to completely obliterate the fistula, then surgery is a valid and safe alternative. Often, these fistulas may seem complex on a quick review of the angiogram, but once the anatomy is clarified and the site of actual "fistulization" is identified, the surgical strategy becomes quite straightforward. Gamma Knife surgery has a limited role in the treatment of DAVFs presenting with hemorrhage. Such DAVFs have a very aggressive natural history after a first hemorrhage ${ }^{4}$ with substantial risks of rehemorrhage during the latency period. Moreover, it has been our anecdotal experience that GKS is less effective in treating DAVFs in locations other than the transverse/sigmoid sinuses and indirect cavernous sinus fistulas. However, in one of our cases, GKS was used as an adjunct to other therapeutic modalities.

In our series, $19 \%$ of patients had residual fistulas at the end of their treatment. This group of patients is quite heterogeneous and includes patients who refused further treatment, patients with very minor and slow residual filling for whom a "wait and see" approach was considered 


\section{J. Daniels et al.}

reasonable, cases ( 2 patients) for which treatment was not considered because of intrinsic risks and/or poor clinical condition, and cases for which further treatment is planned. The role of incomplete therapy is unknown, and we believe that complete angiographic obliteration should be the goal of treatment especially in patients presenting with hemorrhage from a DAVF. However, no hemorrhages at follow-up were observed in our patients who had incomplete obliteration after treatment or those who did not undergo treatment because of refusal or poor initial clinical condition.

\section{Conclusions}

Hemorrhage from DAVFs is often associated with cortical venous drainage and venous varices. There is a striking male predominance in our and other series, and the most common type was tentorial, further suggesting regional differences between DAVF locations between males and females. Treatment strategies that use different treatment modalities and techniques and are based on the clinical and angiographic features are highly effective and safe in the modern management of ruptured intracranial DAVFs. Furthermore, clinical outcomes were good despite patients presenting with intracranial hemorrhage.

\section{Disclosure}

Dr. Lanzino is a consultant for ev3/Covidien. The other authors have no disclosures.

Author contributions to the study and manuscript preparation include the following. Conception and design: Lanzino, Zipfel Acquisition of data: Daniels, Vellimana. Analysis and interpretation of data: Lanzino, Daniels, Vellimana. Drafting the article: Lanzino, Daniels. Critically revising the article: all authors. Reviewed submitted version of manuscript: all authors. Approved the final version of the manuscript on behalf of all authors: Lanzino. Study supervision: Lanzino, Zipfel.

\section{References}

1. Brown RD Jr, Wiebers DO, Nichols DA: Intracranial dural arteriovenous fistulae: angiographic predictors of intracranial hemorrhage and clinical outcome in nonsurgical patients. $\mathbf{J}$ Neurosurg 81:531-538, 1994

2. Choi JH, Mast H, Sciacca RR, Hartmann A, Khaw AV, Mohr JP, et al: Clinical outcome after first and recurrent hemorrhage in patients with untreated brain arteriovenous malformation. Stroke 37:1243-1247, 2006
3. Cognard C, Gobin YP, Pierot L, Bailly AL, Houdart E, Casasco A, et al: Cerebral dural arteriovenous fistulas: clinical and angiographic correlation with a revised classification of venous drainage. Radiology 194:671-680, 1995

4. Duffau H, Lopes M, Janosevic V, Sichez JP, Faillot T, Capelle L, et al: Early rebleeding from intracranial dural arteriovenous fistulas: report of 20 cases and review of the literature. J Neurosurg 90:78-84, 1999

5. Geibprasert S, Pereira V, Krings T, Jiarakongmun P, Toulgoat F, Pongpech S, et al: Dural arteriovenous shunts: a new classification of craniospinal epidural venous anatomical bases and clinical correlations. Stroke 39:2783-2794, 2008

6. King WA, Martin NA: Intracerebral hemorrhage due to dural arteriovenous malformations and fistulae. Neurosurg Clin $\mathbf{N}$ Am 3:577-590, 1992

7. Lasjaunias P, Chiu M, ter Brugge K, Tolia A, Hurth M, Bernstein M: Neurological manifestations of intracranial dural arteriovenous malformations. J Neurosurg 64:724-730, 1986

8. Lawton MT, Sanchez-Mejia RO, Pham D, Tan J, Halbach VV: Tentorial dural arteriovenous fistulae: operative strategies and microsurgical results for six types. Neurosurgery 62 (3 Suppl 1):110-125, 2008

9. Malik GM, Pearce JE, Ausman JI, Mehta B: Dural arteriovenous malformations and intracranial hemorrhage. Neurosurgery 15:332-339, 1984

10. Mitsuhashi Y, Aurboonyawat T, Pereira VM, Geibprasert $\mathrm{S}$, Toulgoat F, Ozanne A, et al: Dural arteriovenous fistulas draining into the petrosal vein or bridging vein of the medulla: possible homologs of spinal dural arteriovenous fistulas. Clinical article. J Neurosurg 111:889-899, 2009

11. Piippo A, Niemelä M, van Popta J, Kangasniemi M, Rinne J, Jääskeläinen JE, et al: Characteristics and long-term outcome of 251 patients with dural arteriovenous fistulas in a defined population. Clinical article. J Neurosurg 118:923-934, 2013

12. Puffer RC, Daniels DJ, Kallmes DF, Cloft HJ, Lanzino G: Curative Onyx embolization of tentorial dural arteriovenous fistulas. Neurosurg Focus 32(5):E4, 2012

13. Satoh K, Satomi J, Nakajima N, Matsubara S, Nagahiro S: Cerebellar hemorrhage caused by dural arteriovenous fistula: a review of five cases. J Neurosurg 94:422-426, 2001

14. Singh V, Smith WS, Lawton MT, Halbach VV, Young WL: Risk factors for hemorrhagic presentation in patients with dural arteriovenous fistulae. Neurosurgery 62:628-635, 2008

Manuscript submitted January 21, 2013.

Accepted April 2, 2013.

Please include this information when citing this paper: DOI: 10.3171/2013.4.FOCUS1335.

Address correspondence to: Giuseppe Lanzino, M.D., Department of Neurological Surgery, Mayo Clinic, Rochester, Minnesota 55905. email: lanzino.giuseppe@mayo.edu. 